JOURNAL WATCH

\title{
Pediatric Tuberculosis
}

Pediatric Infectious Disease (2019): 10.5005/jp-journals-10081-1111

\section{Source: Peter J Dodd, Courtney M Yuen, Mercedes C Becerra, Paul Revill, Helen E Jenkins, James A Seddon. Potential effect of household contact management on childhood tuberculosis: A Mathematical Modelling Study. Lancet Glob Health. 2018;6: e1329-1338. doi:10.1016/S2214-09X(18)30401-7}

The investigators in this mathematical modeling study address the role of household contact management (HCM) for tuberculosis and its potential to prevent substantial morbidity and mortality in children, with impact-for-effort that compares favorably with other interventions.

Using the data, they provide the first global and national estimates of the impact of moving from zero to full coverage of household contact management (with and without preventive therapy for TST-positive children older than 5 years). They have assembled data on tuberculosis notifications, household structure, household contact coprevalence of tuberculosis disease and infection, the efficacy of preventive therapy, and the natural history of childhood tuberculosis. They have used a model to estimate households visited, children screened, and treatment courses are given for active and latent tuberculosis.

They have estimated that full implementation of household contact management would prevent 1,59,500 ( $75 \%$ uncertainty interval [UI] 1,47,000-1,70,900) cases of tuberculosis and 1,08,400 (75\% UI 98,800-1,16,700) deaths in children younger than 15 years (representing the loss of $73,05,000$ [75\% UI 66,63,000-78,74,000] life-years). This study estimates that preventing one child death from tuberculosis would require visiting 48 households, screening 77 children, giving 48 preventive therapy courses, and giving two tuberculosis treatments versus no household contact management.

Household contact management could substantially reduce childhood disease and death caused by tuberculosis globally. The low global coverage (even in India) of this effective intervention needs to increase; funding and implementation research to enable this should be prioritized.

\section{Source: Jenkins HE, Yuen CM. The Burden of Multidrug-resistant Tuberculosis in Children. Int J Tuberc Lung Dis. 2018 May 01;22(5):3-6. doi:10.5588/ijtld.17.0357}

The investigators review current estimates of the global incidence of pediatric MDR-TB disease. They then combine pediatric MDR-TB treatment data from the World Health Organization and recently published case fatality ratio estimates for children with TB to produce mortality estimates for children with MDR-TB. Finally, they combine treatment data and estimates of household size and disease risk to estimate how many children could be treated for presumptive MDR-TB by carrying out household contact investigations around adult MDR-TB patients.

In this study, they find that between 25,000 and 32,000 children develop MDR-TB disease annually, accounting for around 3\% of all pediatric TB cases. Only 3-4\% of these children likely receive MDR-TB treatment. They estimate that around $22 \%$ of children developing the MDR-TB disease will die. They estimate that carrying out household contact investigations around adult MDR-TB patients could find 12 times as many pediatric MDR-TB cases as are currently being identified.

Large gaps exist in our knowledge of the pediatric MDR-TB epidemic. This lack of knowledge both reflects and perpetuates the lack of attention on children with MDR-TB. To save lives, TB programs must prioritize finding and treating children with MDR-TB. To help TB programs understand the magnitude of potentially missed cases, current reporting systems should be revised to capture age-disaggregated data on patients treated for MDR-TB. Moreover, including specific information on children in reports on the global MDR-TB epidemic would help to highlight global and regional treatment gaps. The collection and use of increasingly granular data have been key to previous disease elimination programs and is now needed to reduce morbidity and mortality due to pediatric MDR-TB.

\section{Source: Guiastrennec B, Ramachandran G, Mats O, Karlsson et al. Suboptimal Antituberculosis Drug Concentrations and Outcomes in Small and HIV-Coinfected Children in India: Recommendations for Dose Modifications. Clin Pharmacol Ther. 2018 October;104(4):733-741. doi: 10.1002/cpt.987}

India recently updated its national tuberculosis treatment guidelines and is now moving toward a once-daily dosing regimen. The optimal doses of first-line drugs for all children, in India or elsewhere, has not been firmly established.

This study tries to find out the subpopulations at risk of unfavorable treatment outcomes (therapy failure, death), and what modifications can be made to the new once-daily Indian pediatric tuberculosis treatment guidelines to reduce this risk in these subgroups. This study shows that low rifampin exposures were linked to an increased probability of unfavorable treatment outcome. Rifampin exposure was the lowest in children with low body weight or HIV coinfection.

Clinical practice in India is evolving and this work provides vital information to inform dosing guidelines of the future. More specifically, the study simulations suggest that higher antituberculosis drug dose levels in Indian children, especially those with low body weight or HIV coinfection, can potentially prevent treatment failure or death. This study established dose-exposure-response relationship could be pivotal in the development of future pediatric tuberculosis treatment guidelines.

(c) The Author(s).2019 Open Access This article is distributed under the terms of the Creative Commons Attribution 4.0 International License (https://creativecommons. org/licenses/by-nc/4.0/), which permits unrestricted use, distribution, and non-commercial reproduction in any medium, provided you give appropriate credit to the original author(s) and the source, provide a link to the Creative Commons license, and indicate if changes were made. The Creative Commons Public Domain Dedication waiver (http://creativecommons.org/publicdomain/zero/1.0/) applies to the data made available in this article, unless otherwise stated. 


\section{Source: Deshpande D, Srivastava S, Bendet P, Martin KR, Cirrincione KN, Lee PS, Pasipanodya JG, Dheda K, Gumbo T. 2018. Antibacterial and sterilizing effect of benzylpenicillin in tuberculosis. Antimicrob Agents Chemother 62:e02232-17. https://doi.org/10.1128/AAC.02232-17}

Benzylpenicillin was tried on cultures of Mycobacterium tuberculosis by Fleming and others, but it was found to be ineffective by most. In this study, the investigators found that $>64 \mathrm{mg} /$ liter of static benzylpenicillin concentrations killed significant concentrations of extracellular and intracellular M. tuberculosis over 7 days.

When they added the beta-lactamase inhibitor avibactam, benzylpenicillin maximal kill (Emax) of extracellular log-phase-growth $M$. tuberculosis was significant at a 50\% effective concentration (EC50), while for intracellular M. tuberculosis also it was significant.

The median penicillin (plus avibactam) MIC against South African clinical M. tuberculosis strains ( $80 \%$ either multidrug or extensively drug-resistant) was $2 \mathrm{mg} /$ liter. They mimicked human-like benzylpenicillin and avibactam concentration-time profiles in the hollowfiber model of tuberculosis (HFS-TB). The percent time above the MIC was linked to effect, with an optimal exposure of $>65 \%$. At optimal exposure in the HFS-TB, the bactericidal activity in log phase-growth M. tuberculosis was significant for both extra- and intracellular $M$. tuberculosis and the significant $\mathrm{CFU} / \mathrm{mL}$ were killed over 3 weeks.

In an 8-week HFS-TB study of nonreplicating persistent $M$. tuberculosis, penicillin-avibactam alone and the drug combination of isoniazid, rifampin, and pyrazinamide both killed significant CFU/mL of mycobacteria.

Monte Carlo simulations of 10,000 preterm infants with disseminated disease identified an optimal dose of 10,000 U/ kg (of body weight)/h, while for pregnant women or nonpregnant adults with pulmonary tuberculosis the optimal dose was $25,000 \mathrm{U} / \mathrm{kg} / \mathrm{h}$, by continuous intravenous infusion.

They conclude by saying that penicillin-avibactam should be examined for effect in pregnant women and infants with drug-resistant tuberculosis, to replace injectable ototoxic and teratogenic second-line drugs.

\section{Vikram S Kumar}

Associate Professor and Unit Head, Senior Consultant

Department of Pediatrics

Subbaiah Institute of Medical Sciences

Shivamogga, Karnataka, India

Unity Center for Advanced Pediatric Care

Shivamogga, Karnataka, India 